\title{
Spinning versus Wobbling: How the Brain Solves a Geometry Problem
}

\author{
Jean Laurens, Dominik Strauman, and Bernhard J. Hess \\ Department of Neurology, University of Zurich, CH-8091 Zurich, Switzerland
}

Oscillating an animal out-of-phase simultaneously about the roll and pitch axes ("wobble") changes continuously the orientation of the head relative to gravity. For example, it may gradually change from nose-up, to ear-down, nose-down, ear-down, and back to nose-up. Rotations about the longitudinal axis ("spin") can change the orientation of the head relative to gravity in the same way, provided the axis is tilted from vertical. During both maneuvers, the otolith organs in the inner ear detect the change in head orientation relative to gravity, whereas the semicircular canals will only detect oscillations in velocity (wobble), but not any rotation at constant velocity (spin). Geometrically, the whole motion can be computed based on information about head orientation relative to gravity and the wobble velocity. We subjected monkeys (Macaca mulatta) to combinations of spin and wobble and found that the animals were always able to correctly estimate their spin velocity. Simulations of these results with an optimal Bayesian model of vestibular information processing suggest that the brain integrates gravity and velocity information based on a geometrically coherent three-dimensional representation of head-in-space motion.

\section{Introduction}

The perception of self-orientation and motion in space plays an important role in motor control of balance and locomotion. Over the last decade, evidence has accumulated that motor control involves central representations of "internal models" of movements (Ito, 1989; Kawato, 1999; Davidson and Wolpert, 2005). Many modeling studies on spatial orientation and navigation also suggest that the brain maintains an internal estimate of threedimensional motion in space, which is constantly updated to match afferent information from the vestibular, visual, and proprioceptive sensory systems (Oman, 1982; Droulez and Darlot, 1989; Glasauer, 1992; Merfeld et al., 1993; Merfeld, 1995a,b; Bos and Bles, 2002; Zupan et al., 2002; MacNeilage et al., 2008; Green and Angelaki, 2010). In a similar framework, Laurens and Droulez $(2007,2008)$ have recently proposed that the brain uses Bayesian inference to select the optimal estimate of the actual motion state.

Angular accelerations of the head are detected by the ampullary hair cells of the semicircular canals in the inner ear. However, these inertial sensors cannot detect prolonged rotations at constant velocity. If this rotation occurs about a tilted axis, the otolith organs do detect the body's change in orientation relative to gravity [off-vertical axis rotation (OVAR)] (see Fig. $1 B$ ). Primates

\footnotetext{
Received Nov. 7, 2010; revised April 10, 2011; accepted April 12, 2011.

Author contributions: J.L. designed research; J.L. performed research; J.L., D.S., and B.J.H. analyzed data; J.L., D.S., and B.J.H. wrote the paper.

This work has been supported by the Zurich Center for Integrated Human Physiology (University of Zurich), The Betty and David Koetser Foundation for Brain Research, and the Swiss National Science Foundation. We thank Carla Bettoni, Jakob Thomassen, and Urs Scheifele for their assistance.

Correspondence should be addressed to Jean Laurens, Vestibulo-0culomotor Laboratory Zurich, Department of Neurology, Zurich University Hospital, Frauenklinikstrasse 26, CH-8091 Zurich, Switzerland. E-mail: jean.laurens@gmail.com.

DOI:10.1523/JNEUROSCI.5900-10.2011

Copyright $\odot 2011$ the authors $\quad 0270-6474 / 11 / 318093-09 \$ 15.00 / 0$
}

and certain other species have developed mechanisms to efficiently use the vestibular afferent signals for gaze stabilization through vestibulo-ocular reflexes (VORs) (Guedry, 1965; Young and Henn, 1975; Raphan et al., 1981; Cohen et al., 1983; Harris, 1987; Hess and Dieringer, 1990; Hess and Angelaki, 1993; Angelaki and Hess, 1996a,b; Kushiro et al., 2002). Pertinent observations suggest that the brain estimates head motion in space by processing the vestibular information based on an internal model of head-in-space motion (Merfeld et al., 1999; Angelaki et al., 2004). However, other authors have proposed that reflex eye movements could simply result from appropriately filtering the otolith afferent signals (Hain, 1986; Raphan and Schnabolk, 1988; Raphan and Sturm, 1991; Schnabolk and Raphan, 1992; Raphan and Cohen, 2002), without recourse to an internal model of motion.

To address this controversy, we have used a motion paradigm similar to one used previously (Schor et al., 1984) (cone motion) (see Fig. $1 B$ ), during which the head (and body) oscillates about the interaural and naso-occipital axes in phase quadrature such that it moves through the same sequence of orientations relative to gravity as during OVAR, yet without any rotation about the longitudinal axis. These oscillations activate the vertical semicircular canals that generate compensatory torsional and vertical eye movements (roll-pitch VOR) (see Fig. 1C). Based on the synergy of vertical semicircular canal and otolith afferent activation, the internal model hypothesis predicts that no horizontal response will be generated during cone motion, in contrast to OVAR. A simple filtering of the otolith afferent signals would directly generate a horizontal VOR in both paradigms. We measured the three-dimensional VOR in rhesus monkeys during both OVAR and cone motion, as well as in combinations of these two paradigms. The results of these experiments were simulated with a Bayesian model. 


\section{Materials and Methods}

Animal preparation and eye movement recording. Four female rhesus monkeys (Macaca mulatta) were chronically prepared with skull bolts for head restraint and dual search coils implanted under the conjunctiva for threedimensional eye movement recording as described in previous studies (Hess, 1990; Angelaki and Hess, 1995b). All procedures conformed to the National Institutes of Health Guide for the Care and Use of Laboratory Animals and were approved by the Veterinary Office of the Canton of Zurich.

Three-dimensional eye positions were measured using magnetic search coils (Robinson, 1963) with an Eye Position Meter 3000 (Skalar). Eye position was calibrated as described by Hess et al. (1992), digitized at a sampling rate of $833.33 \mathrm{~Hz}$ (Cambridge Electronic Design; model 1401 plus) and stored on a computer for off-line data analysis. Eye angular velocity vectors $(\Omega)$ were computed: the three components of $\Omega$ represented the torsional $\left(\Omega_{\text {tor }}\right)$, vertical $\left(\Omega_{\mathrm{ver}}\right)$, and horizontal $\left(\Omega_{\mathrm{hor}}\right)$ angular eye velocity. Slow phase eye velocity was computed by detecting and removing quick phase movements using an algorithm similar to the one published by Holden et al. (1992). In the following, the term "angular eye velocity" is synonymous to "slow phase angular eye velocity."

Experimental setup and stimulation protocols. Monkeys were seated in a primate chair with the head restrained in upright position, so that the lateral semicircular canals were approximately earth-horizontal (stereotactic plane tilted $\sim 15^{\circ}$ nose-down). The primate chair was mounted on a computercontrolled motorized four-axis turntable, which was completely surrounded by a lightproof sphere of $1.6 \mathrm{~m}$ diameter. The inner wall of the sphere was covered with black dots of different sizes. The switching on and off of the inside illumination of the sphere was electronically controlled.

Spin and wobbling motion. In a first series of experiments, we compared the VOR elicited by a conventional OVAR paradigm (spin motion only) (see Fig. $1 B$, top panel) with that caused by a so-called cone motion paradigm (wobbling motion only) (see Fig. $1 B$, bottom panel). The way spin and wobble motions superimpose in our paradigms can best be explained by the following line of thoughts. To generate the OVAR motion, the animal was rotated in a tilted position (tilt angle $\alpha$ ) at constant velocity $\left(\omega_{1}\right)$ about its longitudinal axis (see Fig. $1 \mathrm{~A}$, left, axis I). From the perspective of the animal, this motion changed its orientation relative to gravity or, equivalently, caused the gravity vector to rotate about its longitudinal axis (see Fig. $1 \mathrm{~A}$, right). To induce wobble (Laurens et al., 2008), an additional rotation was superimposed about an earth-vertical axis at a generally different constant velocity $\left(\omega_{2}\right)$ (see Fig. $1 \mathrm{~A}$, left, spacefixed axis II). Since this second rotation was about an axis that was always aligned with gravity, it did not affect the animal's head orientation relative to gravity and therefore it did not modulate the otolith input. In other words, the otolith stimulation was the same during all the experiments presented in this study. From the animal's perspective, the angular velocity vector of this second motion (vector $\omega_{2}$ ) (see Fig. 1A, right) moved parallel to gravity, its tip describing a circle in the head yaw plane (see Fig. $1 A$, right). The roll and pitch component of vector $\omega_{2}$ therefore varied sinusoidally, creating a wobble motion. Note that the angular velocity $\omega_{2}$ has a constant yaw (spin) component that adds or subtracts to the spin velocity $\omega_{1}$ depending on the direction of rotation. Thus, counterrotation about axis II will decrease the total spin velocity and increase the wobble velocity, eventually creating a pure wobble motion (cone), in which the head always faces the same direction in the horizontal plane. This can be visualized in Figure $1 B$ : the first axis of the platform rotates identically in the two paradigms, but the counterrotation of the platform during cone motion maintains the orientation of the head in the horizontal plane constant. Inversely, rotation about axis II in the same direction as about axis I will create a "negative" wobble velocity and increase the spin velocity. The resultant motion has a rotation component along the yaw axis, namely $S=\omega_{1}-\omega_{2} \cdot \cos \alpha$ (spin velocity), and a component $W=\omega_{2} \cdot \sin \alpha$, (wobble velocity). Note that $\omega_{1}$ and $\alpha$ are constant. The spin $(S)$ and the wobble $(W)$ velocity are related to each other as follows:

$$
S=\omega_{1}-W / \tan \alpha
$$

Notice that, during cone motion $\left(\omega_{1}=\omega_{2}\right)$, the head is always facing the same direction in the horizontal plane (see Fig. $1 B$, bottom panel) but the spin velocity of the head is in fact not zero because of the term $1 / \tan \alpha$ $\left(4^{\circ} / \mathrm{s}\right.$ for $\omega_{1}=\omega_{2}=30^{\circ} \%$ s with $\left.\alpha=30^{\circ}\right)$.

OVAR motion was produced by tilting the axis I (see Fig. $1 A$ ) by $\alpha=$ $30^{\circ} \mathrm{off}$ vertical and rotating it at $\omega_{1}=30 \% \mathrm{~s}$ in darkness. Cone motion was obtained by additionally counterrotating the axis II at $\omega_{2}=30 \%$ s. Combinations of these motions were obtained by rotating the axis II at velocities from $-60 \%$ to $105 \%$ in steps of $15 \%$. In each animal, all combinations were tested in clockwise and counterclockwise direction (for which the signs of $\omega_{1}$ and $\omega_{2}$ were reversed). Motion duration was $90 \mathrm{~s}$ for each trial. (Animations of these protocols can be found at http://www.vertigocenter.ch/laurens/SpinWobble.html.)

Spin stimulations during OVAR. To further investigate the interactions between spin and wobble motion in the VOR, we varied the sensory signals indicating a spin motion at the initiation of OVAR. This was done by applying stimuli that either activated the lateral semicircular canals or the horizontal optokinetic system. Initially, all animals were rotated in darkness at a velocity of $\omega^{\prime}=(30-\omega)^{\circ} / \mathrm{s}$ around a vertical axis for $90 \mathrm{~s}$ until the yaw VOR subsided. Then the rotation was rapidly changed to $30 \%$, which elicited a yaw VOR with gain close to 1 . Two seconds after this acceleration step, the animal was tilted toward $30^{\circ}$ off-vertical (in $0.4 \mathrm{~s}$, triangular velocity profile with $180^{\circ} / \mathrm{s}^{2}$ acceleration) while it continued to rotate at $30 \%$ s. In this manner, the induced sensory cues indicated a rotation in yaw (i.e., a spin motion) at any desired velocity $\omega$ when the OVAR was initiated, although the animal was actually spinning at $30 \%$. Alternatively, to activate the visual system, the optokinetic sphere was rotated at $\omega^{\prime}=(30-\omega)^{\circ} / \mathrm{s}$, while the animal rotated at $30 \%$, both around a common vertical axis. During this phase preceding the OVAR, the animal thus rotated relative to the visual surround (i.e., the illuminated sphere) at velocity $\omega$. After $90 \mathrm{~s}$, the light was extinguished and the animal was tilted $2 \mathrm{~s}$ thereafter while continuing the rotation in yaw. The visual stimulation created a horizontal optokinetic nystagmus with a velocity close to $\omega$, followed by an after response [optokinetic afternystagmus (OKAN)] as soon as the light was extinguished. Both protocols were used to initiate OVAR trials with an initial yaw VOR/OKAN of $\omega=$ $-60 \% \mathrm{~s},-30 \% \mathrm{~s}, 0 \% \mathrm{~s}, 30 \% \mathrm{~s}, 60 \%$ s. We also tested the VOR protocol at velocities $\omega= \pm 90^{\circ} / \mathrm{s}$, but not the OKN/OKAN protocol, since these velocities exceeded the saturation velocity. Note that the total time between spin signal induction by vestibular or visual stimulation and completion of tilt was $2.4 \mathrm{~s}$. This delay is considerably shorter that the time constant of the VOR and OKAN; thus, the overlap of spin decay and OVAR onset was negligible. All animals underwent four trials at each of the six (seven) velocities $\omega$. All trials were initiated by tilting the animals through $30^{\circ}$ from upright to either one of the four orientations (i.e., nose-up, nose-down, left ear-down, and right ear-down). OVAR was maintained for $48 \mathrm{~s}$. Since the vestibular and optokinetic results were indistinguishable, we pooled the data for each of the four different head orientations.

Angular eye velocity analysis. The spin and wobble motions generated a constant-velocity horizontal VOR as well as vertical and torsional VOR velocities oscillating sinusoidally at a frequency of $1 / 12 \mathrm{~Hz}$. This frequency corresponded to the rotation relative to gravity at $\omega_{1}=30 \%$ s. In the following, we call the constant horizontal response component spin or yaw VOR, and the oscillating vertical $\left(\Omega_{\mathrm{ver}}\right)$ and torsional $\left(\Omega_{\text {tor }}\right)$ responses wobble VOR. The wobble VOR velocity was computed as follows: during wobbling at a velocity $W$, the head velocity in roll and pitch is $W \cdot(\cos (\theta), \sin (\theta))$. In this equation, $\theta$ is the orientation of the head relative to gravity [i.e., the component of gravity in the yaw plane of the head (nose-down, $\theta=0$; right ear-down, $\theta=-90^{\circ}$; nose-up, $\theta=-180^{\circ}$; left ear-down, $\theta=-270^{\circ}$; notice that $\theta$ decreases during clockwise rotation of the head relative to gravity, since this rotation causes gravity to rotate counterclockwise relative to the head)]. Thus, for ideal compensation, the torsional and vertical VOR velocities should be $\Omega_{\text {tor }}=$ $-W \cdot \cos \theta$ and $\Omega_{\mathrm{ver}}=-W \cdot \sin \theta$. We computed the amplitude of the vector $\left(\Omega_{\text {tor }}, \Omega_{\text {ver }}\right)$ as well as its phase relative to the ideal VOR (i.e., the velocity and the phase of the wobble VOR). Angular eye velocity during combinations of spin and wobble motion (see Fig. 2C) was averaged over the last $48 \mathrm{~s}$ of stimulation. The angular eye velocity in Figure $3 D$ was averaged using a sliding window with a width of $\pm 0.5 \mathrm{~s}$.

We estimated the translational VOR during OVAR by an approach first used by Merfeld et al. (1999) (see Fig. 5) (see Results). According to 
A

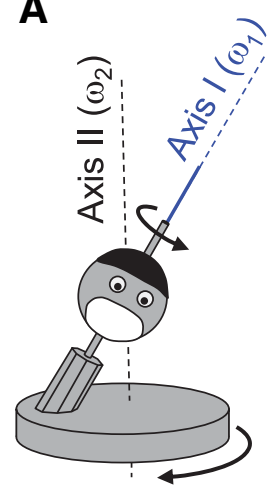

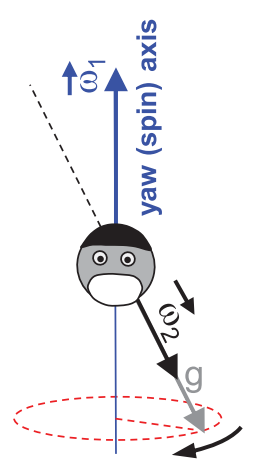

Pitch, roll

(wobble) plane
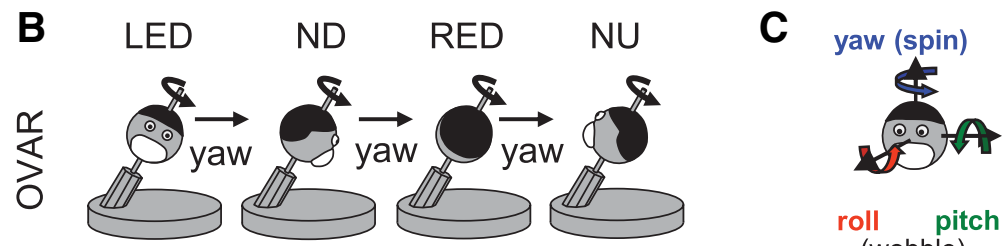

roll pitch (wobble)

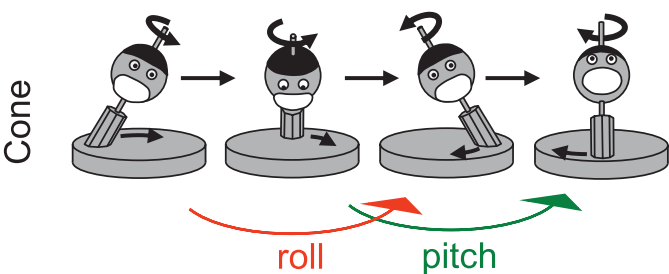

Horizontal (spin VOR)

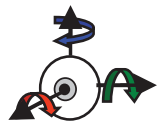

torsional vertical (wobble VOR)

Figure 1. Illustration of the OVAR and cone motion protocol. $A$, Left, Schematic representation of the rotator used in the experiments. In all protocols, the subject was rotated about axis I at a constant velocity $\left(\omega_{1}\right)$. Simultaneous rotation about axis II generated a motion that is called wobble motion. Cone motion is generated when $\omega_{1}=\omega_{2}$, as in $\boldsymbol{B}$. $\boldsymbol{A}$, Right, Representation of $\left(\omega_{1}\right)$, $\left(\omega_{2}\right)$, and the gravity vector $g$ in an egocentric frame of reference. $\boldsymbol{B}$, Illustration of OVAR and cone motion paradigms. Top, In the OVAR paradigm, the head continuously changes its orientation relative to gravity while the subject rotates in tilted position at constant velocity about its longitudinal axis (LED, left ear-down; ND, nose-down; RED, right ear-down; NU, nose-up). Bottom, In the cone motion paradigm, the head changes its orientation relative to gravity in a similar way because of $90^{\circ}$ out-of-phase pitch and roll oscillations. Thereby the subject faces always the same direction in the horizontal plane. Notice that the motion in both panels differs only by the counterrotation of the rotator. $\boldsymbol{C}$, Representation of the three principal axes of head and eye movements. Curvilinear arrows indicate positive directions of head movements (head scheme) and eye movements (eye scheme).

A

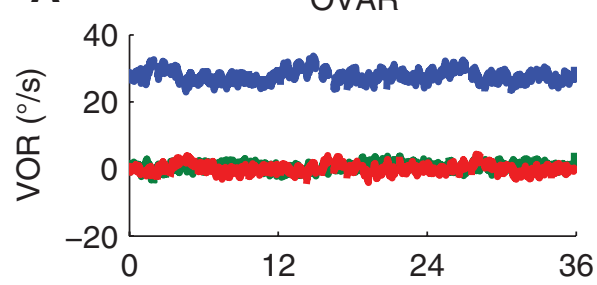

B Cone

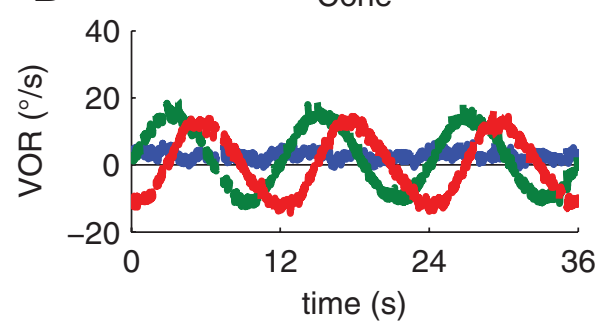

C

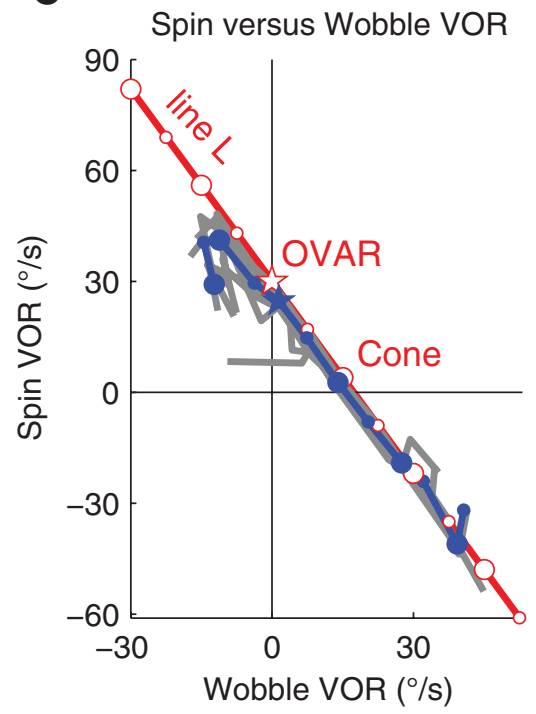

rens and Droulez (2007, 2008). This model computes an optimal estimate of head and body motion in space by assigning a probability to "every" possible motion in space - in fact, the model only considers plausible motions in space, which are selected by a sampling process called particle filtering (Maskell and Gordon, 2002). The probability depends on how well the sensory afferent signals that would be generated by a particular motion correspond to the actually received afferent signals, as well as to an a priori probability distribution of motions. The model assumes that the noise in the semicircular canals afferent signals limits the accuracy of the information provided about head angular velocity. In particular, the gain of the canals is zero during constantvelocity rotation, and therefore there is no information about steady-state yaw or spin velocity during OVAR and the cone motion paradigm. In other words, all possible spin velocities are equally compatible with the afferent information of the semicircular canals. In contrast, the brain receives relatively accurate information about the wobble velocity, since wobble motion includes angular accelerations that efficiently activate the semicircular canals. Therefore, the probability of a motion estimate that deviates from the wobble velocity sensed by the semicircular canals is low. The model also assumes

this method, the yaw VOR during OVAR can be considered as a translational and a rotational VOR. Since gravity modulates sinusoidally along the interaural axis, the contribution of a possible translational VOR must be modulated in a sinusoidal manner as a function of head orientation, in contrast to the rotational VOR. We recorded the yaw VOR during OVAR trials, in which the animals were initially tilted toward nose-up, nosedown, left ear-down, and right ear-down. By averaging these trials, a contribution of the translational VOR is nullified, leaving a response representing the rotational VOR. Then, by subtracting the rotational VOR from the yaw VOR, we obtained the translational VOR for each trial. By comparing trials at different head orientations, we estimated the influence of head orientation on the translational VOR at each point in time.

Bayesian modeling. The experimental results were compared with simulations performed with an optimal Bayesian model as described by Lau- that low translational accelerations are a priori more likely. Head motion relative to gravity and linear accelerations are sensed by the otolith organs, whose signals $(F)$ are related to gravity $(G)$ and linear head acceleration $(A)$ according to the following: $F=G-A$. The a priori probability on linear accelerations tends to favor motion estimates for which $A=G-F$ is small [i.e., for which the estimate of head orientation relative to gravity $(G)$ fully accounts for the afferent signals $(F)$ ]. In the process of Bayesian inference, each possible motion in space conforms to geometric constraints, and the orientation of the head in space (i.e., relative to gravity) is computed by integrating the estimated angular velocity over time. The prior on linear acceleration indirectly promotes rotation estimates that correspond to a rotation of the head relative to gravity as sensed by the otoliths. At the same time, the model assigns a high a priori probability to low angular velocities. This causes a small 

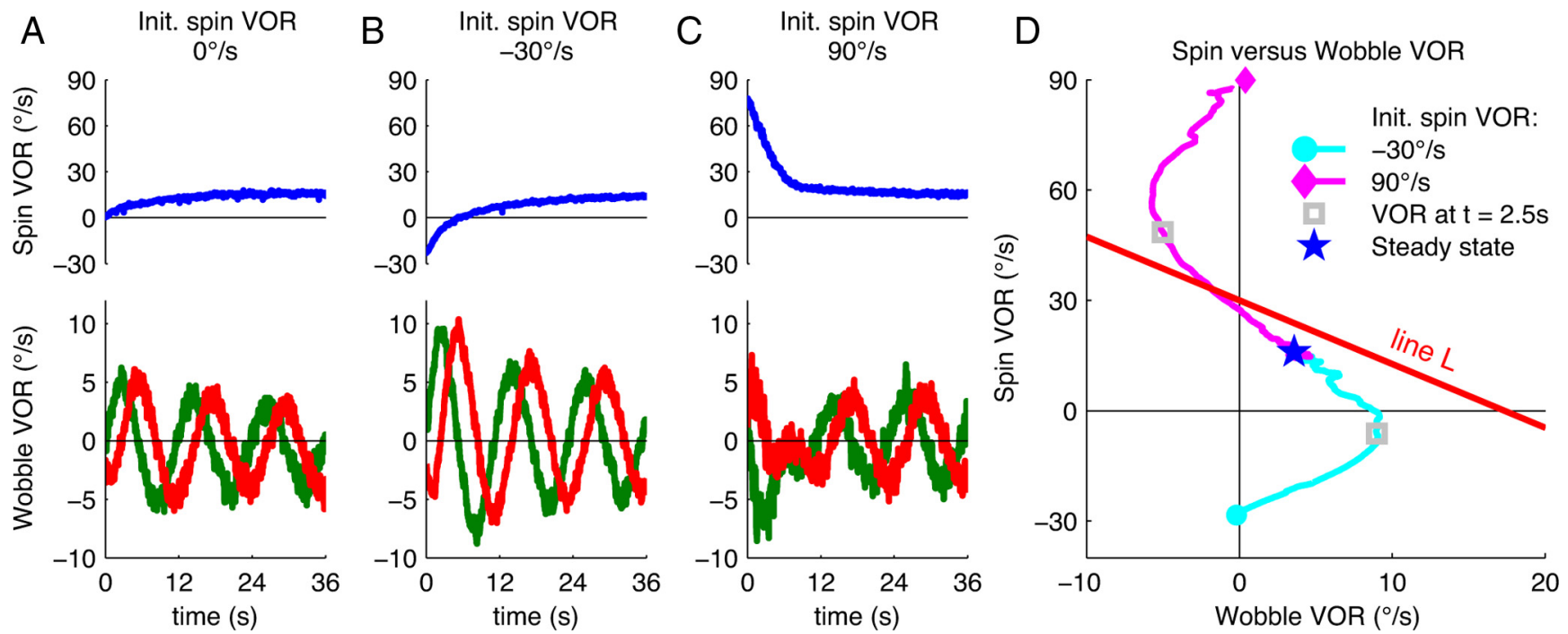

Figure 3. Transient responses to spin pulse-injections before OVAR onset. $A$, Spin and wobble VOR during the first three cycles of OVAR initiated without any spin signal. $B, C$, Spin and wobble VOR during OVAR after injection of a negative $(\boldsymbol{B})$ or positive $(\boldsymbol{C})$ spin. $\boldsymbol{D}$, Spin as a function of wobble showing an $\mathrm{S}$-shaped behavior after an initial spin VOR of $-30^{\circ} / \mathrm{s}$ (blue circle and trace) or after an initial spin VOR of $90^{\circ} / \mathrm{s}$ (diamond and magenta trace). Gray square, VOR at $t=2.5 \mathrm{~s}$. Blue star, Average steady-state VOR. For comparison, also line L is plotted (as in Fig. 2C). The traces in $\boldsymbol{A}-\boldsymbol{D}$ are averages across all trials.

bias, which, however, plays a minor role in modeling the present paradigms. Finally, it is assumed that the visual-optokinetic pathways carry head velocity information relative to the environment with a certain signal-to-noise ratio that allows reproducing the phenomena of optokinetic nystagmus and afternystagmus (Laurens and Droulez, 2007). We used the same model parameters as in the study by Laurens and Droulez (2008) (i.e., $\sigma_{\Omega}=40 \% \mathrm{~s}, \sigma_{\mathrm{V}}=10 \%, \sigma_{\mathrm{O}}=7 \%$ s, except for $\sigma_{\mathrm{A}}=1 \mathrm{~m} / \mathrm{s}^{2}$, which we adapted to the present experiments).

\section{Results}

\section{Spin and wobble conventions}

We have studied combinations of spin and wobble motion, which moved the head through the same orientations relative to gravity. The spin motion consisted of a constant-velocity rotation around the yaw axis (Fig. 1C), whereas the wobble motion consisted of oscillations around the pitch and roll axes (Fig. 1C). We measured the three-dimensional VOR during these paradigms (Fig. 1C, bottom panel). To be compensatory for a spin motion, the VOR should consist in constant-velocity horizontal eye movements ("spin VOR"), whereas for wobble motion, it should exhibit oscillatory vertical and torsional eye movements ("wobble VOR").

\section{Angular eye velocity during the steady-state motion}

In a first experiment, we tested whether the brain can correctly interpret the sensory signals during OVAR and cone motion. During OVAR, the head was rotating at a constant velocity of $30 \%$ around the yaw axis (i.e., it was spinning). We found that monkeys exhibited a spin VOR (Fig. $2 A$ ), with an average velocity of $24 \pm 2.5 \%$ s (mean \pm SD between trials), close to the head velocity. A very small wobble VOR was also measured (1.5 \pm $0.8^{\circ} / \mathrm{s}, 10 \pm 18^{\circ}$ phase lag). During the cone motion, the head was oscillating around the pitch and roll axes (wobble) with peak velocity of $15 \%$ and also rotating in yaw (spinning) at a low velocity of $4 \%$ s (Eq. 1). We found a wobble VOR that closely matched the head wobble motion in phase and amplitude $\left(13.8 \pm 0.7^{\circ} / \mathrm{s}, 3 \pm 5^{\circ}\right.$ phase lag), whereas the spin VOR was $2.7 \pm$ $0.5^{\circ}$ s (wobble VOR) (Fig. $2 B$ ). This result was in line with our hypothesis that the spin VOR is based on an internal model of motion in space.
We tested this hypothesis further by submitting the animals to various combinations of spin and wobble motion, whose velocities are linked by Equation 1 (see Materials and Methods). Accordingly, each velocity combination represents a point on the line $\mathrm{L}$ in the spin-wobble plane (Fig. 2C). All of these combinations represent the same motion of the head relative to gravity. Therefore, the motion of the head relative to gravity as sensed by the otoliths during these experiments was geometrically consistent with any point located on line L. Finally, the semicircular canals directly detected the wobble component of the motion, whereas the brain received no direct sensory information about the spin velocity. We found that the eye movements measured during these combinations closely corresponded to the head velocity provided the spin velocity remained $<30 \%$ s. This was true even at the level of individual trials (Fig. $2 C$, gray lines). Outside of this range, the angular velocity of the VOR deviated from head velocity, suggesting that the central motion integration pathways reached their saturation point (Angelaki et al., 2000). This corroborates previous findings that the brain estimates head-in-space motion by using a geometrically correct model of head motion, in which the estimate of angular velocity matches head motion relative to gravity (Angelaki and Hess, 1995b).

\section{Transient responses to spin stimulation}

According to our working hypothesis, the internal estimate of head rotation should match the head orientation relative to gravity as sensed by the otoliths. Geometrically, this implies that the velocities of the spin and wobble VOR should closely correspond to Equation 1, represented by the line $\mathrm{L}$ in Figure $2 C$. In the first series of experiments, we studied the VOR during steady-state motion. In this condition, the lateral semicircular canals do not provide any information about the spin velocity. This velocity could nevertheless be computed as a function of the wobble velocities detected by the vertical semicircular canals as indicated by Equation 1. In a second series of experiments, we tested the hypothesis that sensory stimuli indicating a spin should reciprocally influence the wobble VOR according to Equation 1. At the beginning of OVAR, we therefore applied transient stimuli indicating a spin motion at various velocities (see Materials and Methods, 
Spin stimulations during OVAR) to test the hypothesis whether the animals would develop an internal estimate of wobble motion in an attempt to match the motion estimate predicted by Equation 1 (Fig. 2C, see line $\mathrm{L}$ ).

A first version of these experiments consisted in initiating OVAR without the usual preceding step-like activation of the semicircular canals. In this condition, the spin VOR took several seconds to develop (Fig. 3A). During this period, we observed a wobble VOR that decayed while the spin VOR built up [in line with previous reports (Hess and Angelaki, 1999)]. Interestingly, we could amplify this effect by adding a negative step in spin rotation immediately before the onset of OVAR (see Materials and Methods). The initially negative spin VOR quickly reversed and reached the same steady-state level as during a normal OVAR trial (Fig. 3B). Simultaneously, the initial wobble VOR was enhanced and subsequently decreased as the spin VOR redeveloped. These phenomena are summarized in Figure 3D: Starting from an initial condition in which the spin VOR was $-30 \%$ s and the wobble VOR zero (circle on Fig. 3D), the rapidly developing wobble VOR did bring the eye movements in $\sim 2.5$ s close to the theoretically expected line L (gray square). Then both the spin and wobble VOR converged toward a steady state (star). In this phase, we observed a clear linear relationship between the spin and wobble VOR, as predicted by Equation 1. Finally, we applied positive spin velocity steps in a series of trials to induce a strong positive spin VOR. In the first few seconds of these paradigms, the wobble VOR showed an inverse phase relationship compared with the previous conditions (Fig. $3 C$ ). This reversed VOR can be described as a negative wobble VOR (Fig. 3D) that also brought the eye velocity close to the expected line L. Subsequently, the spin and wobble VOR converged along a straight line toward steady state (star). Note that a certain degree of habituation occurred during these experiments. When an OVAR trial was performed in the first experiment (Fig. $2 A, C$ ), the steady-state VOR reached an average of $24 \%$ spin VOR and $1.5 \%$ wobble VOR. In this second series of experiment, the steady state reached $20 \%$ spin and $2.5 \%$ s wobble VOR when the initial spin VOR was close to the real motion of the head (i.e., $30 \%$ ). In other conditions (Fig. 3A-C), the steady-state VOR reached an average of $16.5^{\circ} \mathrm{s}$ spin and $3.5 \%$ sobble, indicating that processing large spin signals induced an additional habituation. Although the habituation affected the steady state to a certain extent, the linear relationship between spin and wobble VOR during OVAR was preserved.

The line formed by the spin and wobble VOR had a similar orientation as the line $\mathrm{L}$ described by Equation 1 and it intersected the ordinate at about the same point. This suggests that it is indeed the kinematics represented by Equation 1 that governs the brain's adjustment of wobble velocity estimation (Fig. 3D, line L). However, although the experimental trajectory approached the line L, its slope was steeper (Fig. 3D, line L). In other words, the estimated wobble VOR was only one-half in amplitude as that predicted by Equation 1. Since there was no real wobble velocity of the head in this particular case, the estimated wobble that developed in an attempt to match the estimated motion of the head with the otolith signal was in conflict with the absence of semicircular canal signals. We will interpret this lower amplitude as a trade-off between minimizing two mismatches below in Principle of Bayesian estimation.

\section{Bayesian modeling}

We used the Bayesian model (Laurens and Droulez, 2007, 2008) to compute the statistically optimal estimate of motion during
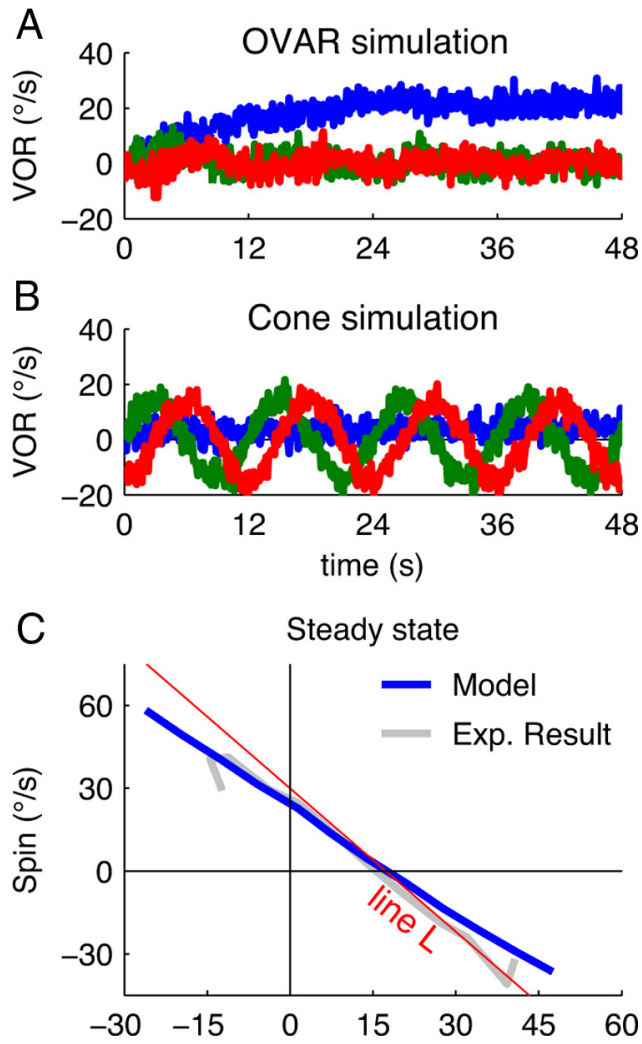

D Init VOR

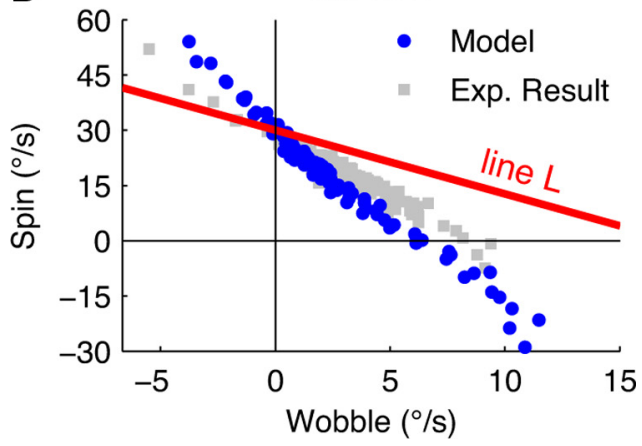

Figure 4. Bayesian model simulations. A, OVAR paradigm (compare with Figs. $2 A, 3 A$ ). $\boldsymbol{B}$, Cone motion paradigm (compare with Fig. $2 B$ ). C, Simulated steady-state responses to combinations of spin and wobble motion (blue line) and comparison with the experimental results (as in Fig. 2C. D, Simulated transient responses to injection of spin pulses (as in Fig. 3D).

our experimental paradigms. During OVAR, the motion estimate obtained from the simulated OVAR evolved toward a steady state, for which the optimal estimate was spin rotation (Fig. 4A). During cone motion, the simulation produced an estimate of oscillations in pitch and roll (i.e., a wobble motion) (Fig. $4 B$ ), closely corresponding to the experimental findings (Fig. 2A,B). We simulated various combinations of spin and wobble motion and computed the spin and wobble responses based on optimal motion estimation. We found that these responses formed a line close to the line L predicted by Equation 1 (Fig. 4C), in good agreement with experimental results. Moreover, during the first few seconds of the simulated OVAR response, we observed a wobble response (Fig. $4 A$ ) as during the experiments (Fig. $3 A$ ). Simulating OVAR trials with various initial spin amplitudes (Fig. $4 D$ ), we found that the estimated wobble was modulated as a function of initial spin velocity, although it was lower than pre- 
dicted by Equation 1, in agreement with the experimental findings. Together, the simulations based on the Bayesian model could accurately reproduce a number of important aspects of our results.

\section{Linear acceleration estimation}

So far, the interpretation of our results was based on the hypothesis that head motion relative to gravity is faithfully reported by the otolith organs. Head motion relative to gravity was the same in all our protocols and corresponded to combinations of spin and wobble motion according to Equation 1 (Figs. $2 C, 3 D, 4 C, D$, line L). Thus, we considered that the otolith information was in fact sufficient for the brain to know that the motion of the head corresponded to a motion according to line L. However, the otoliths are in fact sensitive to both the gravity vector $(G)$ and linear acceleration $(A)$ experienced during head translations, according to the equation $F=G-A$, in which $F$ is the gravito-inertial acceleration that activates the otoliths. Therefore, although the animals were never translated in our experiment, the brain could have interpreted the variations of the otolith signals as the result of some incident linear accelerations of the head. A number of studies have shown that the brain, confronted by this ambiguity, tends to interpret the otolith signal as the consequence of gravity and minimize the linear acceleration estimate (Graybiel, 1952; Guedry, 1974; Paige and Seidman, 1999). Therefore, our assumption that the brain attempts to develop a motion estimate that is coherent with head motion, as sensed by the otoliths, was a justified simplification. However, the internal model hypothesis predicts that deviation of the angular motion estimate from the predicted line L (Eq. 1) should result in a mismatch between the internal representation of gravity and the incoming otolith signals and lead to a nonzero estimate of translational head acceleration.

We tested this hypothesis by adopting a method used previously by Merfeld et al. (1999). Linear acceleration along the interaural axis leads to a horizontal translational VOR that should superimpose to a rotational VOR with amplitude modulated by head orientation relative to gravity. At the frequency used in our experiment $[30 \%$ s (i.e., $0.083 \mathrm{~Hz}$ motion of the head relative to gravity)], this VOR should be in phase with the estimated acceleration of the head (Paige and Tomko, 1991). We illustrate this method by assuming that an animal is rotating in a counterclockwise direction. In an egocentric frame of reference, the gravity vector rotates clockwise around the animal's body vertical axis. If the estimate of gravity leads the actual head orientation (re gravity), then the translational VOR will take the form of rightward eye movements when the animals are in a nose-up position (Fig. $5 A$ ) and leftward movements in nose-down position (Fig. 5B). By summing up with the rightward spin VOR, the translational VOR should cause the total horizontal VOR to peak in nose-up orientation (Fig. 5A).

During the second experiment, we induced spin velocity signals at various velocities right before initiating the OVAR. In some conditions, these signals induced a spin VOR faster than $30 \%$. We pooled the results whenever the spin VOR fell in between $40 \%$ and $60 \%$ s (i.e., $50 \%$ on average) and analyzed the modulation of the horizontal VOR as a function of head orienta-

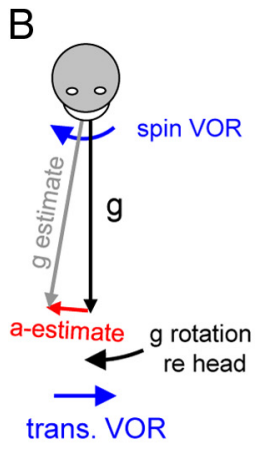

\section{Translational VOR and a-estimate as a function of spin VOR}
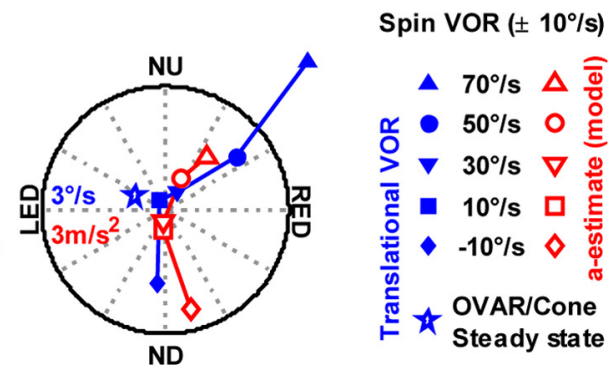

Figure 5. Translational VOR induced by in injection of spin stimuli before OVAR onset. $\boldsymbol{A}, \boldsymbol{B}$, Illustration of the acceleration estimate ( $a$-estimate) generated when the estimated gravity ( $g$-estimate) leads the actual gravity vector $(g)$, during counterclockwise rotation of the head, and of the superposition of the translational and angular (spin) VOR. C, Gain and phase versus head model (red), as a function of the spin VOR. The phase of the simulated translational acceleration is inverted to account for opposite direction.

tion (see Materials and Methods). We found that the VOR was faster under this condition in an orientation between nose-up and right ear-down (Fig. 5C, circle). This result was consistent with the hypothesis that the overestimation of the spin velocity caused the estimated gravity vector to lead the actual head orientation, causing in turn a translational VOR. In a symmetric manner, the VOR peaked when the animals reached a nose-down orientation if the spin VOR was slower than $30 \%$ s. Simulating the estimation of acceleration in these conditions with the Bayesian model, we found that the phase of the simulated estimate of interaural acceleration perfectly corresponded to the translational VOR measured in the experiments. In contrast, the average translational VOR during steady-state OVAR and cone motion remained close to zero (Fig. 5C, star).

\section{Principle of Bayesian estimation}

The Bayesian model computes the probability distribution of three-dimensional motion over time. This high-dimensional process is difficult to visualize. However, it can be simplified by reformulating the inference in terms of spin and wobble motion. The OVAR stimulus consists only of spin motion about the $z$-axis (Fig. 6A), whereas the cone motion consists only of a wobble motion. In between these extremes, there is a continuum of intermediate motion states (Fig. $6 \mathrm{~B}$, mixture of $10 \%$ wobble and $12.7^{\circ} \mathrm{s}$ spin motion). In this simplified version, we assume that the model "knows" that the rotation is a combination of spin and wobble motion. Bayesian inference consists in computing the probability of all these combinations by combining three probability distributions. The first distribution is the a priori concerning the most likely distribution of angular velocities, which favors velocities close to zero (Fig. $6 A, B$, first column). The second is derived from the a priori about the distribution of translational accelerations, which favors low accelerations. Since motion estimates that contradict Equation 1 lead to an estimation of translational acceleration, this a priori favors motion estimates that are close to line L (second column). Finally, the third distribution comes from the semicircular canals, which can detect oscillations corresponding to the actual wobble motion with a certain uncertainty but never rotations at constant velocity as the spin in our experiments (i.e., the uncertainty on spin velocity is infinite). Therefore, the distribution of possible velocities according to the canals scatters around a vertical line W (third column, green line). The two lines L and W in Figure 6 intersect at one point that corresponds to the real velocity of the head. The multiplication of 
A

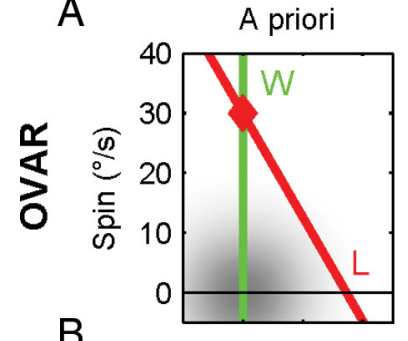

$\mathrm{B}$

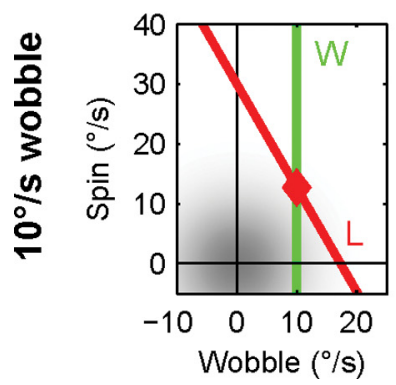

Otolith signal
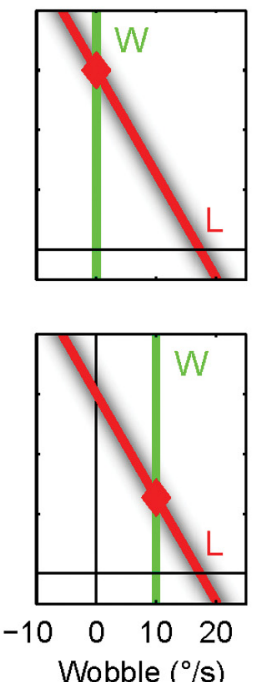
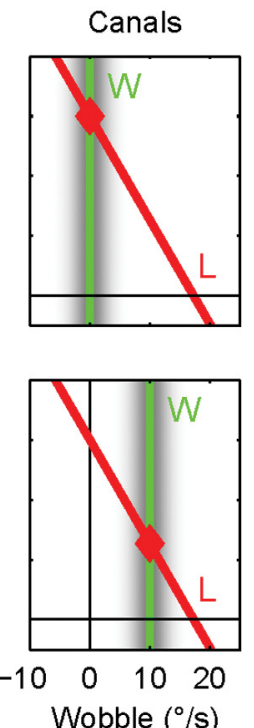

Steady-state
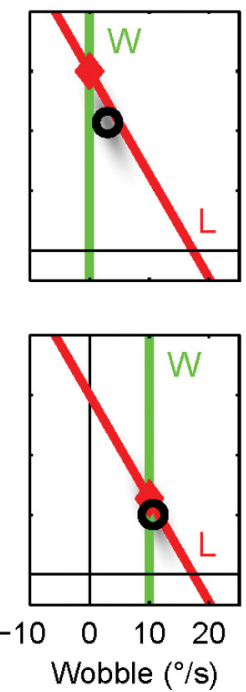

C

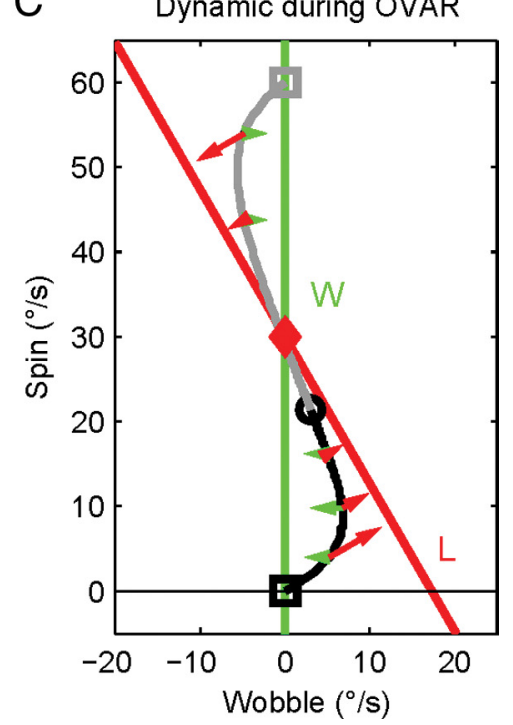

Figure 6. Principles of Bayesian inference. The intensity plots represent the probability distributions according to the a priori, otolith and semicircular canal information in $\boldsymbol{A}$ for OVAR and in $\boldsymbol{B}$ for combinations of spin and wobble motion. The steady-state distribution (last column) is the product of the a priori distribution of angular velocity (first column) and the information provided by the otoliths and the canals (second and third columns). The line L corresponds to Equation 1 as in the previous figures; the line W corresponds to the wobble velocity of the head. Red diamonds, Real motion of the head; black circles, the final estimate (last column). C, Evolution of the estimate over time during OVAR, starting from an initial value of zero (black square, line) or $60^{\circ} / \mathrm{s}$ spin (gray square, line). The arrows indicate the influence of canals and otoliths on the estimation at various points in time.

the probability distributions according to the semicircular canal and otolith signals would lead to a final estimate centered at this intersection point. However, because of the uncertainty about these signals, the a priori knowledge influences the final estimate by shifting it somewhat toward zero (i.e., downward and rightward). This reduces the total velocity of the final estimate while maintaining it close to the line L. These examples illustrate how one can compute the steady-state velocity estimate using Bayesian inference. In addition, the time evolution of motion estimation during a spin-wobble paradigm can be obtained as follows: suppose, for instance, that the subject undergoes an OVAR motion (30\% spin and no wobble) and consider a motion estimate corresponding to a certain position in the spin-wobble phase space (Fig. $6 C$, open squares). The effect of otolith signals will be to drive it toward the line $\mathrm{L}$ (no head translation, red arrows), whereas the effect of semicircular canal signals will be to attract it toward the ordinate axis (green arrows) since there is no wobble velocity during OVAR. Finally, the a priori information (not represented) will attract it toward the origin of the graph. Starting from a spin of $0 \%$ s (black square) or $60 \%$ s (gray square), the motion estimate quickly converges toward a line where the influence of the semicircular canal and otolith information is balanced and drive it slowly toward the final estimate (black circle). This process corresponds to the experimental measurements and to the simulations realized with the three-dimensional Bayesian model (Fig. 3D).

\section{Discussion}

We have demonstrated that the brain estimates head orientation in space in the cone motion paradigm as well as in its variants with surprising accuracy. One rather suggestive way to explain this capacity is that the brain makes use of a geometrically appropriate three-dimensional internal model that represents motion in space. As shown in previous studies, this type of reconstruction of motion in space is only feasible if the brain takes into account position and velocity information from both the otolith and the semicircular canals (Hess and Angelaki, 1993; Angelaki and Hess, 1995b; Angelaki et al., 1999; Merfeld et al., 1999; Green et al., 2005). To push this analysis one step further, we applied velocity steps of various amplitudes about the yaw axis while the animal was rotating in yaw at constant velocity in tilted position. These maneuvers induced conflicting sensory signals about the spin motion. To maintain geometrical consistency between the central rotation estimates and the head motion relative to gravity, we found that the animals developed an estimate of wobble motion, although they were in fact not wobbling. Yet, being in conflict with the missing modulation of vertical semicircular canal activity, the wobble estimate was lower than the amount necessary for perfect consistency with head motion relative to gravity. We used the Bayesian model to simulate the trade-off between geometrical consistency and sensory conflict, and found that the measured eye movements were close to the optimal solution. Finally, the internal model hypothesis predicted that the deviation from the estimated head motion relative to gravity and the actual gravitoinertial signals from the otolith organs should result in a nonzero estimate of translation. Indeed, the observed horizontal response can be interpreted as a translational VOR.

Our results support the notion that the brain integrates information about three-dimensional rotation velocity into a coherent estimate of head position in space. This estimate can be used to extract the inertial and gravitational components of the otolith afferent signals, in agreement with previous studies (Angelaki et al., 1999, 2002, 2004; Merfeld et al., 1999). Our analysis suggests that conflicting motion signals are resolved by a process of optimal estimation, similar as shown in numerous previous studies (Ernst and Banks, 2002; Weiss et al., 2002; MacNeilage et al., 2007; Angelaki et al., 2009; Fetsch et al., 2009) as well as in modeling work on vestibular information processing (Laurens et al., $2007,2008)$. By demonstrating that the brain effectively uses geometrically consistent three-dimensional representations, this study supports the notion that the internal model hypothesis as formulated in the general context of motor control (Ito, 1989; Kawato, 1999; Davidson and Wolpert, 2005) is also an important 
concept for understanding how the brain solves spatial orientation problems.

\section{Previous work on the internal model hypothesis}

Oman (1982) originally proposed that motion sickness arises when the brain fails to match an internal representation of motion with the sensory afferent inflow. This hypothesis inspired three-dimensional modeling such as the work of Droulez and Darlot (1989), which generates optimal motion estimates by using a gradient ascent procedure to minimize cost functions. The functions are designed in such a way that they favor estimates that are both geometrically consistent and match sensory inputs. Thus, the principles are the same as those underlying the Bayesian model. Later models by Merfeld (Merfeld et al., 1993; Merfeld, 1995a,b), Glasauer (Glasauer and Merfeld, 1997), and Zupan (Zupan et al., 2002) implemented the internal model in a computationally more efficient ad hoc architecture, which compares the expected sensory inputs with the effective afferent inflow. Any mismatch is used to correct the estimate of motion, which is computed according to an internal model of three-dimensional motion. Feedback loops ensure that the estimate is conforming to the sensory inflow (for analyses and review of this framework, see also Bos and Bles, 2002; MacNeilage et al., 2008; Laurens and Angelaki, 2011). These loops can also generate a trade-off between different sources of sensory information when they are in conflict. All these models have been shown to successfully reproduce eye movements induced by off-vertical axis rotations.

Although the Bayesian model implements basically similar ideas as these previous models, the crucial difference is that it performs the optimal estimation directly based on two simple assumptions: (1) the existence of an appropriate internal model of motion and (2) the existence of some measures quantifying or estimating the relative reliability of the various sensory inputs (e.g., based on the amount of noise in the afferent channels and possibly additional other factors). Thus, the critical advantage of using the Bayesian approach over previous models is that it allows one to test directly whether or not behavior can be explained by simple rules of optimal three-dimensional self-motion processing, as originally proposed by Oman (1982).

\section{Other models of vestibular information processing}

As an alternative to the internal model hypothesis, other authors (Hain, 1986; Raphan and Sturm, 1991; Angelaki, 1992; Raphan and Cohen, 2002) have proposed that the spin VOR measured during OVAR is produced by filtering otolith signals. However, these models predict that the otolith activation during cone motion, being identical with the activation during OVAR, should produce the same spin VOR, in contradiction with our results. Additionally, these studies focused on the spin response during OVAR, disregarding the wobble component that is pronounced at the onset of OVAR and cannot easily be accounted for by these models. To reproduce the present experimental results, these models would require substantial modifications, among which the addition of a specific mechanism that accurately cancels the spin VOR during cone motion. Although this is of course possible, for instance by adapting the gravity-dependent velocity storage mechanism proposed by Raphan and Cohen (2002), it would make these models conceptually much more complex than the Bayesian approach, which is based on the internal model hypothesis. Furthermore, all the results of the experiments considered here agree perfectly well with the output of an optimal motion estimator. Therefore, adapting previous filtering models to these results would be equivalent to suggesting that the brain performs optimal estimation of head motion through a computationally and conceptually complex filtering process rather than by using an internal model.

\section{Neural implementation}

A variety of studies have addressed the issue of neural substrates that might be involved in constructing an internal model of selfmotion in space. It has been demonstrated that the spin (yaw) VOR during OVAR is abolished after lesions of the nodulus and uvula (Angelaki and Hess, 1995a), suggesting that the cerebellum plays a crucial role in building an internal model of selforientation and motion. Such models seem to play an important role in many sensorimotor transformations (Ito, 1989, 2006; Davidson and Wolpert, 2005; Green and Angelaki, 2010) as well as possibly in Bayesian inference (Paulin, 2005). Along these lines, Angelaki et al. (2004) have demonstrated that brainstem and cerebellar neurons integrate rotation information over time to extract the acceleration component from the otolith input. A realistic neural network architecture that integrates angular velocity in three dimensions was recently proposed by Green et al. (2005).

\section{Conclusion}

Previous studies (Angelaki et al., 1999, 2004; Merfeld et al., 1999) showed that the brain uses angular motion information to disambiguate the otolith signals. Here, we created and tested a new family of motion paradigms and demonstrated that the brain produces motion estimates that are geometrically consistent and can accurately be simulated by Bayesian inference.

\section{Notes}

Supplemental material for this article is available at http://www.vertigocenter.ch/ laurens/SpinWobble.html. We present a set of animated movies that illustrate the motion paradigms used in our experiments. This material has not been peer reviewed.

\section{References}

Angelaki DE (1992) Detection of rotating gravity signals. Biol Cybern 67:523-533.

Angelaki DE, Hess BJ (1995a) Lesion of the nodulus and ventral uvula abolish steady-state off-vertical axis otolith response. J Neurophysiol 73: $1716-1720$.

Angelaki DE, Hess BJ (1995b) Inertial representation of angular motion in the vestibular system of rhesus monkeys. II. Otolith-controlled transformation that depends on an intact cerebellar nodulus. J Neurophysiol 73:1729-1751.

Angelaki DE, Hess BJ (1996a) Three-dimensional organization of otolithocular reflexes in rhesus monkeys. I. Linear acceleration responses during off-vertical axis rotation. J Neurophysiol 75:2405-2424.

Angelaki DE, Hess BJ (1996b) Three-dimensional organization of otolithocular reflexes in rhesus monkeys. II. Inertial detection of angular velocity. J Neurophysiol 75:2425-2440.

Angelaki DE, McHenry MQ, Dickman JD, Newlands SD, Hess BJ (1999) Computation of inertial motion: neural strategies to resolve ambiguous otolith information. J Neurosci 19:316-327.

Angelaki DE, Merfeld DM, Hess BJ (2000) Low-frequency otolith and semicircular canal interactions after canal inactivation. Exp Brain Res 132:539-549.

Angelaki DE, Newlands SD, Dickman JD (2002) Inactivation of semicircular canals causes adaptive increases in otolith-driven tilt responses. J Neurophysiol 87:1635-1640.

Angelaki DE, Shaikh AG, Green AM, Dickman JD (2004) Neurons compute internal models of the physical laws of motion. Nature 430:560-564.

Angelaki DE, Klier EM, Snyder LH (2009) A vestibular sensation: probabilistic approaches to spatial perception. Neuron 64:448-461.

Bos JE, Bles W (2002) Theoretical considerations on canal-otolith interaction and an observer model. Biol Cybern 86:191-207.

Cohen B, Suzuki JI, Raphan T (1983) Role of the otolith organs in genera- 
tion of horizontal nystagmus: effects of selective labyrinthine lesions. Brain Res 276:159-164.

Davidson PR, Wolpert DM (2005) Widespread access to predictive models in the motor system: a short review. J Neural Eng 2:S313-S319.

Droulez J, Darlot C (1989) The geometric and dynamic implications of the coherence constraints in three-dimensional sensorimotor interactions. In: Attention and performance XIII (Jeannerod M, ed), pp 495-526. New York: Erlbaum.

Ernst MO, Banks MS (2002) Humans integrate visual and haptic information in a statistically optimal fashion. Nature 415:429-433.

Fetsch CR, Turner AH, DeAngelis GC, Angelaki DE (2009) Dynamic reweighting of visual and vestibular cues during self-motion perception. J Neurosci 29:15601-15612.

Glasauer S (1992) Interaction of semicircular canals and otoliths in the processing structure of the subjective zenith. Ann N Y Acad Sci 656:847-849.

Glasauer S, Merfeld DM (1997) Modelling three-dimensional vestibular responses during complex motion stimulation. In: Three-dimensional kinematics of eye, head and limb movements (Fetter M, Haslwanter T, Misslisch H, eds), pp 387-398. Amsterdam: Harwood Academic.

Graybiel A (1952) Oculogravic illusion. AMA Arch Ophthalmol 48:605615.

Green AM, Angelaki DE (2010) Internal models and neural computation in the vestibular system. Exp Brain Res 200:197-222.

Green AM, Shaikh AG, Angelaki DE (2005) Sensory vestibular contributions to constructing internal models of self-motion. J Neural Eng 2:S164-S179.

Guedry FE Jr (1965) Orientation of the rotation axis relative to gravity: its influence on nystagmus and the sense of rotation. Acta Otolaryngol 60:30-48.

Guedry FE (1974) Psychophysics of vestibular sensation. In: Handbook of sensory physiology, Vol VI (Kornhuber HH, ed), pp 1-154. New York: Springer.

Hain TC (1986) A model of the nystagmus induced by off vertical axis rotation. Biol Cybern 54:337-350.

Harris LR (1987) Vestibular and optokinetic eye movements evoked in the cat by rotation about a tilted axis. Exp Brain Res 66:522-532.

Hess BJ (1990) Dual-search coil for measuring 3-dimensional eye movements in experimental animals. Vision Res 30:597-602.

Hess BJ, Angelaki DE (1993) Angular velocity detection by head movements orthogonal to the plane of rotation. Exp Brain Res 95:77-83.

Hess BJ, Angelaki DE (1999) Oculomotor control of primary eye position discriminates between translation and tilt. J Neurophysiol 81:394-398.

Hess BJ, Dieringer N (1990) Spatial organization of the maculo-ocular reflex of the rat: responses during off-vertical axis rotation. Eur J Neurosci 2:909-919.

Hess BJ, Van Opstal AJ, Straumann D, Hepp K (1992) Calibration of threedimensional eye position using search coil signals in the rhesus monkey. Vision Res 32:1647-1654.

Holden JR, Wearne SL, Curthoys IS (1992) A fast, portable desaccading program. J Vestib Res 2:175-179.

Ito M (1989) Long-term depression. Annu Rev Neurosci 12:85-102.

Ito M (2006) Cerebellar circuitry as a neuronal machine. Prog Neurobiol 78:272-303.

Kawato M (1999) Internal models for motor control and trajectory planning. Curr Opin Neurobiol 9:718-727.

Kushiro K, Dai M, Kunin M, Yakushin SB, Cohen B, Raphan T (2002) Compensatory and orienting eye movements induced by off-vertical axis rotation (OVAR) in monkeys. J Neurophysiol 88:2445-2462.

Laurens J, Angelaki DE (2011) The functional significance of velocity storage and its dependence on gravity. Exp Brain Res 210:407-422.
Laurens J, Droulez J (2007) Bayesian processing of vestibular information. Biol Cybern 96:389-404.

Laurens J, Droulez J (2008) Bayesian modeling of visuo-vestibular interactions. In: Probabilistic reasoning and decision making in sensory-motor systems (Bessière P, Laugier C, Siegwart R, eds), pp 291-313. New York: Springer.

Laurens J, Hess BJ, Straumann D (2008) Geometrical considerations on canal-otolith interactions during OVAR and Bayesian modelling. Prog Brain Res 171:287-290.

MacNeilage PR, Banks MS, Berger DR, Bülthoff HH (2007) A Bayesian model of the disambiguation of gravitoinertial force by visual cues. Exp Brain Res 179:263-290.

MacNeilage PR, Ganesan N, Angelaki DE (2008) Computational approaches to spatial orientation: from transfer functions to dynamic Bayesian inference. J Neurophysiol 100:2981-2996.

Maskell S, Gordon N (2002) A tutorial on particle filters for on-line nonlinear/non-gaussian bayesian tracking. IEEE Trans Signal Process 50:174-188.

Merfeld DM (1995a) Modeling human vestibular responses during eccentric rotation and off vertical axis rotation. Acta Otolaryngol Suppl 520:354-359.

Merfeld DM (1995b) Modeling the vestibulo-ocular reflex of the squirrel monkey during eccentric rotation and roll tilt. Exp Brain Res 106: 123-134.

Merfeld DM, Young LR, Oman CM, Shelhamer MJ (1993) A multidimensional model of the effect of gravity on the spatial orientation of the monkey. J Vestib Res 3:141-161.

Merfeld DM, Zupan L, Peterka RJ (1999) Humans use internal models to estimate gravity and linear acceleration. Nature 398:615-618.

Oman CM (1982) A heuristic mathematical model for the dynamics of sensory conflict and motion sickness. Acta Otolaryngol Suppl 392:1-44.

Paige GD, Seidman SH (1999) Characteristics of the VOR in response to linear acceleration. Ann N Y Acad Sci 871:123-135.

Paige GD, Tomko DL (1991) Eye movement responses to linear head motion in the squirrel monkey. I. Basic characteristics. J Neurophysiol 65:1170-1182.

Paulin MG (2005) Evolution of the cerebellum as a neuronal machine for Bayesian state estimation. J Neural Eng 2:S219-S234.

Raphan T, Cohen B (2002) The vestibulo-ocular reflex in three dimensions. Exp Brain Res 145:1-27.

Raphan T, Schnabolk C (1988) Modeling slow phase velocity generation during off-vertical axis rotation. Ann N Y Acad Sci 545:29-50.

Raphan T, Sturm D (1991) Modeling the spatiotemporal organization of velocity storage in the vestibuloocular reflex by optokinetic studies. J Neurophysiol 66:1410-1421.

Raphan T, Cohen B, Henn V (1981) Effects of gravity on rotatory nystagmus in monkeys. Ann N Y Acad Sci 374:44-55.

Robinson DA (1963) A method of measuring eye movement using a scleral search coil in a magnetic field. IEEE Trans Biomed Eng 10:137-145.

Schnabolk C, Raphan T (1992) Modeling 3-D slow phase velocity estimation during off-vertical-axis rotation (OVAR). J Vestib Res 2:1-14.

Schor RH, Miller AD, Tomko DL (1984) Responses to head tilt in cat central vestibular neurons. I. Direction of maximum sensitivity. J Neurophysiol 51:136-146.

Weiss Y, Simoncelli EP, Adelson EH (2002) Motion illusions as optimal percepts. Nat Neurosci 5:598-604.

Young LR, Henn VS (1975) Nystagmus produced by pitch and yaw rotation of monkeys about non-vertical axes. Fortschr Zool 23:235-246.

Zupan LH, Merfeld DM, Darlot C (2002) Using sensory weighting to model the influence of canal, otolith and visual cues on spatial orientation and eye movements. Biol Cybern 86:209-230. 